\title{
Early evaluation of radiation-induced parotid damage in patients with nasopharyngeal carcinoma by T2 mapping and mDIXON Quant imaging: initial findings
}

Nan Zhou ${ }^{1+}$, Chen Chu ${ }^{1+}$, Xin Dou ${ }^{1}$, Weibo Chen ${ }^{2}$, Jian He ${ }^{1 *}$, Jing Yan ${ }^{3^{*}}$, Zhengyang Zhou ${ }^{1^{*}}$ and Xiaofeng Yang ${ }^{4}$

\begin{abstract}
Background: Radiation-induced parotid damage is a common complication in patients with nasopharyngeal carcinoma (NPC) treated with radiotherapy to head and neck region, which severely reduce the life quality of those patients. The aim of this study was to early evaluate the changes of irradiated parotid glands with T2 mapping and mDIXON Quant imaging.

Methods: Forty-one patients with NPC underwent conventional magnetic resonance imaging for nasopharynx and neck, and T2 mapping and mDIXON Quant imaging for bilateral parotid glands within 2 weeks before radiotherapy (pre-RT), 5 weeks after the beginning of radiotherapy (mid-RT), and 4 weeks after radiotherapy (post-RT). Parotid volume, T2 values, fat fraction (FF) values, and mean radiation dose were recorded and analyzed.

Results: From pre-RT to mid-RT, parotid volume decreased (atrophy rate, $27.0 \pm 11.5 \%$ ), while parotid T2 and FF values increased (change rate, $6.0 \pm 6.2 \%$ for T2 value and $9.1 \pm 9.9 \%$ for FF value) significantly. From mid-RT to post-RT, parotid T2 value continuously increased (change rate, $4.6 \pm 7.7 \%$ ), but parotid FF value decreased (change rate, $-9.9 \pm 18.2 \%$ ) significantly. Change rate of parotid T2 value significantly correlated with parotid atrophy rate from pre-RT to post-RT $(r=0.313, P=0.027)$. Multiple linear regression analysis showed that parotid $\mathrm{T} 2$ value (standardized coefficient $[S C]=-0.259, P=0.001)$ and FF value $(S C=-0.320, P=0.014)$ negatively correlated with parotid volume, while parotid T2 value positively correlated with MR scan time point $(S C=0.476, P=0.001)$ significantly. Parotid T2 and FF values showed excellent reproducibility (intraclass correlation coefficient, 0.935-0.992).
\end{abstract}

Conclusions: T2 mapping and mDIXON Quant imaging is useful for noninvasive evaluation of radiation-induced parotid damage.

Keywords: Radiation-induced parotid damage, Magnetic resonance imaging, T2 mapping, mDIXON Quant imaging, Quantitative evaluation

\section{Background}

Patients with nasopharyngeal carcinoma (NPC) always suffer from radiation-induced xerostomia, dysphagia, and even dental caries, which severely reduce their life quality [1]. In recent years, intensity-modulated radiotherapy

\footnotetext{
*Correspondence: hjxueren@126.com; yj20030610@126.com;

zyzhou@nju.edu.cn

${ }^{+}$Equal contributors

'Department of Radiology, Nanjing Drum Tower Hospital, The Affiliated Hospital of Nanjing University Medical School, Nanjing 210008, China

${ }^{3}$ The Comprehensive Cancer Centre of Nanjing Drum Tower Hospital, Clinical

College of Nanjing Medical University, Nanjing 210008, China

Full list of author information is available at the end of the article
}

(IMRT) has been applied to treat NPC, which helps to alleviate radiation-induced parotid damage [2]. Due to their sensitivity to radiation [3], parotid glands cannot entirely escape radiation-induced damage even with IMRT [4]. Since parotid glands are the largest salivary glands producing $60 \%-65 \%$ of the whole saliva [5], early evaluation of radiation-induced parotid damage would facilitate a timely adjustment of treatment scheme to alleviate the damage of parotid glands.

Degree of xerostomia can be clinically evaluated with Radiation Therapy Oncology Group (RTOG) criteria [6], which mainly relies on subjective symptoms. Biopsy can 
bring microstructural information of irradiated parotid glands [7], but it is invasive and unsuitable for clinical practice. Scintigraphy can quantitatively assess the changes of parotid function [8], which has a disadvantage of extra radiation exposure. Magnetic resonance (MR) imaging is a noninvasive modality to evaluate radiation-induced parotid damage with a high soft tissue contrast [9].

Previous studies confirmed the glandular edema and fatty replacement in irradiated parotid glands $[10,11]$. Tissue edema can be quantitatively evaluated by T2 mapping, since T2 relaxation time extends when free water increases [12]. Fat content in tissues can be quantified with fat fraction (FF) generated by mDIXON Quant imaging [13]. The feasibility of T2 mapping and mDIXON Quant imaging in parotid glands has been confirmed in healthy volunteers $[14,15]$. Nevertheless, application of those MR imaging has never been reported in evaluating radiationinduce parotid damage during radiotherapy.

Therefore, the primary purpose of this study was to early evaluate the dynamic changes of radiation-induced parotid damage with T2 mapping and mDIXON Quant imaging. Correlations between parotid T2 or FF values and parotid atrophy rate or mean radiation dose were also investigated.

\section{Methods}

\section{Patients}

This study was approved by the institutional review board. After providing written informed consents, 51 patients was enrolled prospectively. The inclusion criteria were: (1) with a biopsy confirmed diagnosis of NPC; (2) scheduled to undergo IMRT; (3) willing to receive MR evaluation and follow-ups in our hospital. The exclusion criteria included: (1) with a history of radiotherapy to the head and neck region $(n=0)$; (2) with parotid gland diseases, such as Sjögren's syndrome $(n=1)$; (3) with MR contraindications, such as cardiac pacemaker or cochlear implants $(n=0)$; (4) unable to accomplish the whole course of MR follow-ups $(n=7)$; (5) with geometric distortion or physiologic motion on MR images $(n=2)$. Hence, 41 patients with NPC served as our study cohort (male, 29; female, 12; age range, 21-70 years; mean age, $49.1 \pm 11.5$ years).

\section{Chemoradiotherapy and xerostomia degree assessment}

The chemoradiotherapy scheme for NPC patients has been described in a previous article [16]. All patients received MR scans at three time points: pre-RT, within 2 weeks before radiotherapy; mid-RT, 5 weeks after the beginning of radiotherapy; and post-RT, 4 weeks after radiotherapy. Bilateral parotid glands were analyzed separately due to different radiation doses received by them. Parotid mean radiation dose was obtained from the treatment planning systems of TomoTherapy HiArt (TomoTherapy, Madison,
WI, USA) and Pinnacle ${ }^{3}$ (Philips Medical Systems, Fitchburg, WI, USA). The accumulated mean radiation dose of parotid gland at mid-RT $(20.4 \pm 2.7 \mathrm{~Gy})$ or postRT $(29.2 \pm 3.3$ Gy) was also calculated. The mean radiation dose of parotid gland in each patient was under the constraint in our hospital (30-50 Gy for $50 \%$ of parotid volume).

One hour before MR scanning, the xerostomia degree of patients was assessed by a radiation oncologist (X.X., with 10 years' experience in radiotherapy to the head and neck region) according to RTOG criteria [6]. The xerostomia degree was grade 0 in all patients at pre-RT, and increased to grade 1 (20 patients) or 2 ( 21 patients) at mid-RT. From mid-RT to post-RT, the xerostomia degree remained unchanged in 36 patients, and decreased from grade 2 to 1 in 4 patients, and increased from grade 1 to 2 in 1 patients.

\section{MR examination}

All patients was required to fast for at least $2 \mathrm{~h}$ before MR scanning. A 3.0-T MR scanner (Ingenia, Philips Medical Systems, Best, the Netherlands) with a 16-channel head\&neck phased-array coil was used. 32-channel Torso coil was added for mDIXON Quant imaging. The patient's position was head first and supine. Among 41 patients, 22 patients underwent conventional MR and T2 mapping imaging (from May 2016 to January 2017), 6 patients underwent conventional MR, T2 mapping, and mDIXON Quant imaging (from January 2017 to March 2017), and the remaining 13 patients underwent conventional MR and mDIXON Quant imaging (from March 2017 to July 2017), respectively. The conventional MR imaging included fat-saturated T2-weighted imaging in coronal and transverse planes, T1-weighted imaging in coronal, sagittal, and transverse planes, and contrast enhanced fatsaturated T1-weighted imaging in coronal, sagittal, and transverse planes.

T1-weighted imaging was obtained by using turbo spinecho (TSE) sequence, whose parameters were as follows: repetition time (TR) / echo time $(\mathrm{TE})=400-675 \mathrm{msec} /$ $18 \mathrm{msec}$, field of view $(\mathrm{FOV})=22 \mathrm{~cm}$, voxel size $=0.8 \mathrm{~mm} \times$ $0.92 \mathrm{~mm}$, matrix $=276 \times 215$, slice thickness $=5 \mathrm{~mm}$, slices $=38$, number of signals averaged $(\mathrm{NSA})=2$. The scan duration was $2 \min 27 \mathrm{~s}$.

T2 mapping was obtained by using multi-slice multiecho TSE sequence, whose parameters were as follows: $\mathrm{TR} / \mathrm{TE} 1=2109 \mathrm{msec} / 17 \mathrm{msec}$, delta $\mathrm{TE}=17 \mathrm{msec}$, echoes $=5, \mathrm{FOV}=22 \mathrm{~cm}$, voxel size $=1.8 \mathrm{~mm} \times 2.4 \mathrm{~mm}$, matrix $=124 \times 90$, slice thickness $=4 \mathrm{~mm}$, slices $=20$, $\mathrm{NSA}=1$. The scan duration was $33 \mathrm{~s}$.

mDIXON Quant imaging was obtained by using 3D fast field echo (FFE) sequence, whose parameters were as follows: TR / TE1 $=10 \mathrm{msec} / 1.47 \mathrm{msec}$, delta TE $=1.2 \mathrm{msec}$, echoes $=6$, FOV $=25 \mathrm{~cm} \times 24 \mathrm{~cm}$, voxel 
size $=1.2 \mathrm{~mm} \times 1.2 \mathrm{~mm}, \quad$ matrix $=208 \times 201, \quad$ slice thickness $=2.5 \mathrm{~mm}$, slices $=64, \mathrm{NSA}=2$. The scan duration was $2 \min 7 \mathrm{~s}$. A low flip angle of only $3^{\circ}$ was applied to limit the T1 bias, and 6 echoes was used to correct for $\mathrm{T}^{*}$ effects.

\section{Image analysis}

Measurements of MR parameters were performed by two radiologists (X.X. and X.X., with 7 and 15 years' experience in MR imaging of head and neck region, respectively) independently. Both of them were blinded to the clinical data of patients. Parotid volume, T2, and FF values were measured on a workstation (Extended MR WorkSpace 2.6.3.5, Philips Medical Systems, Best, the Netherlands).

Each slice area of parotid gland was measured on T1weighted image. Parotid volume was calculated by using the following equation: $\mathrm{V}=\Sigma \mathrm{S} \times(\mathrm{ST}+\mathrm{SG})$, where $\mathrm{V}$ is parotid volume, $\mathrm{S}$ is each slice area, ST is slice thickness, and SG is slice gap. The atrophy rate of parotid gland was calculated by using the following equation: $R_{V}=\left(V_{\text {pre }}-V_{\text {mid/post }}\right) / V_{\text {pre }} \times 100 \%$, where $R_{V}$ is the atrophy rate of parotid gland from pre-RT to midRT or post-RT, and $V_{\text {pre }}$ and $V_{\text {mid/post }}$ are parotid volume at pre-RT, mid-RT, and post-RT, respectively.

After acquisition of T2 mapping and mDIXON Quant sequence, T2 and FF maps were automatically calculated. The regions of interest (ROIs) were drawn within the largest three slices of parotid gland on T2-wighted image (T2 mapping sequence with $\mathrm{TE}=51 \mathrm{msec}$ ) and FF map to include as much parotid parenchyma as possible excluding visible parotid ducts and retromandibular veins. The ROIs on T2-wighted image were manually copied to the corresponding T2 map to calculate T2 value. The $\mathrm{T} 2$ and $\mathrm{FF}$ values were defined as the averaged value of the largest three slices. The change rates of parotid T2 and FF values were calculated by using the following equation: $R_{\text {Para }}=\left(\mathrm{Para}_{\text {mid } / \text { post }}-\mathrm{Para}_{\text {pre }}\right) /$ Parapre $\times 100 \%$, where $R_{\text {Para }}$ is the change rates of parotid T2 or FF values from pre-RT to mid-RT or post-RT, and Para $_{\text {pre }}$ and Para $m$ mid/post are the T2 or FF value of parotid gland at pre-RT, mid-RT, and post-RT, respectively.

The final T2 and FF values of parotid gland were recorded as the averaged value of the two radiologists' measurements. With an interval of 12 weeks, parotid T2 and FF values were re-measured by the second radiologist to assess the intra-oberver reproducibility.

\section{Statistical analysis}

Continuous data were shown as mean \pm standard deviation. The dynamic changes of parotid volume, T2 and FF values were analyzed by using paired sample $t$ test with Bonferroni correction. Two independent-samples $t$ test was used to compare the change rates of parotid T2 or FF value between men and women. Pearson's correlation test was used to detect correlations between the change rates of $\mathrm{T} 2$ or FF value and parotid atrophy rate, mean radiation dose, or age. Correlations among parotid T2 or FF value, parotid volume, and MR scan time point was detected by multiple linear regression analysis. Intraclass correlation coefficient (ICC) was used to evaluate the reproducibility of parotid $\mathrm{T} 2$ and FF values measurements. All statistical analyses were performed using SPSS 16.0 software (SPSS Inc., Chicago, IL, USA). A $P$ value $<0.017$ for paired sample $t$ test with Bonferroni correction and a $P$ value $<0.05$ for other statistical analyses indicated a statistical significance.

\section{Results}

Dynamic changes of parotid volume, T2 and FF values during radiotherapy

The dynamic changes of parotid volume, T2 and FF values are shown in Table 1 . The T1-weighted images, $\mathrm{T} 2$ and FF maps of bilateral parotid glands in one patient with NPC are shown in Fig. 1.

From pre-RT to mid-RT, parotid volume decreased significantly (atrophy rate, $27.0 \pm 11.5 \%$ ). However, no significant difference was found in parotid volume from mid-RT to post-RT. Hence, the overall atrophy rate of parotid volume from pre-RT to post-RT was $27.0 \pm 10.8 \%$.

From pre-RT to mid-RT, parotid T2 value increased significantly (change rate, $6.0 \pm 6.2 \%$ ), and continued to increase significantly from mid-RT to post-RT (change rate, $4.6 \pm 7.7 \%$ ). Hence, the overall change rate of parotid T2 value from pre-RT to post-RT was $10.8 \pm 10.0 \%$.

From pre-RT to mid-RT, parotid FF value increased significantly (change rate, $9.1 \pm 9.9 \%$ ), and then decreased significantly from mid-RT to post-RT (change rate, $-9.9 \pm 18.2 \%$ ). Hence, parotid FF value did not change significantly from pre-RT to post-RT.

From pre-RT to mid-RT or post-RT, no significant differences of the change rates of parotid T2 or FF value were found between men $(6.1 \pm 6.1 \%$ and $10.1 \pm 11.0 \%$ for $\mathrm{T} 2$ and FF values at mid-RT, $11.5 \pm 10.2 \%$ and -7.4 $\pm 22.7 \%$ for $\mathrm{T} 2$ and $\mathrm{FF}$ values at post-RT, respectively) and women $(5.4 \pm 1.9 \%$ and $11.5 \pm 4.5 \%$ for $\mathrm{T} 2$ and $\mathrm{FF}$ values at mid-RT, $8.0 \pm 5.1 \%$ and $10.0 \pm 5.8 \%$ for $\mathrm{T} 2$ and FF values at post-RT, respectively) (all $P>0.05$ ).

Table 1 Dynamic changes of parotid MR parameters during radiotherapy

\begin{tabular}{llll}
\hline & pre-RT & mid-RT & post-RT \\
\hline$V\left(\mathrm{~cm}^{3}\right)$ & $27.2 \pm 8.2$ & $19.4 \pm 5.5^{\mathrm{a}}$ & $19.5 \pm 5.7^{\mathrm{a}}$ \\
T2 (msec) & $71.7 \pm 5.2$ & $76.0 \pm 7.3^{\mathrm{a}}$ & $79.4 \pm 9.0^{\mathrm{a}, \mathrm{b}}$ \\
$\mathrm{FF}(\%)$ & $38.2 \pm 9.7$ & $42.1 \pm 10.3^{\mathrm{a}}$ & $38.8 \pm 13.5^{\mathrm{b}}$
\end{tabular}

Note: $V$ volume, $F F$ fat fraction, pre- $R T$ within 2 weeks before radiotherapy, mid-RT 5 weeks after the beginning of radiotherapy, post-RT 4 weeks after radiotherapy. ${ }^{\mathrm{a}} P<0.017$ compared with pre-RT and ${ }^{\mathrm{b}} P<0.017$ compared with mid-RT using paired sample $t$ test with Bonferroni correlation 

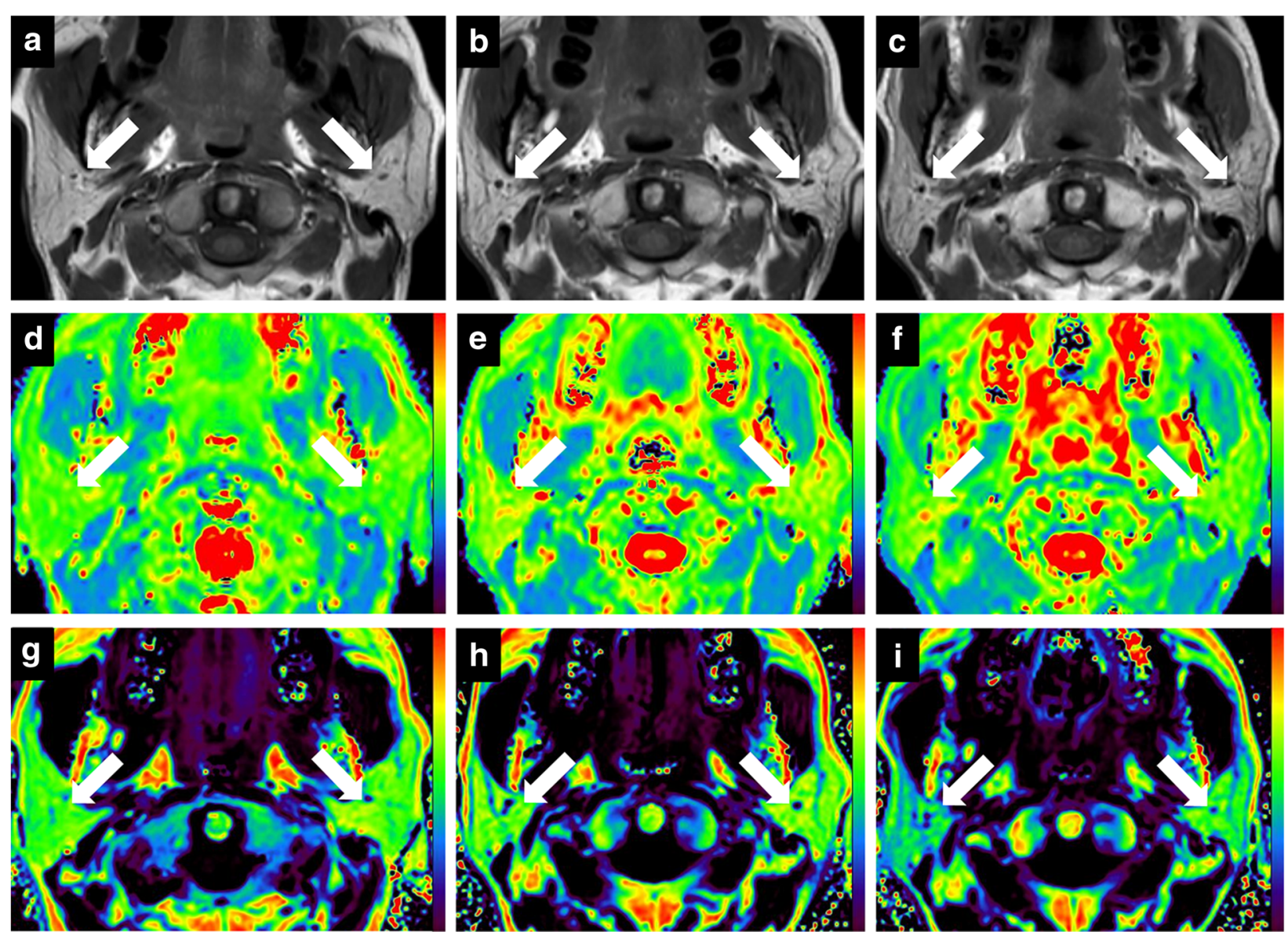

Fig. 1 Dynamic changes of T1-weighted images (a-c), T2 maps (d-f), and fat fraction (FF) (g-i) maps of bilateral parotid glands (white arrows) in one patient with nasopharyngeal carcinoma (NPC) within 2 weeks before radiotherapy (pre-RT), 5 weeks after the beginning of radiotherapy (mid-RT), and 4 weeks after radiotherapy (post-RT), respectively. T1-weighted images show that right and left parotid volume are $30.1 \mathrm{~cm}^{3}$ and $30.2 \mathrm{~cm}^{3}$ (pre-RT, a), $21.9 \mathrm{~cm}^{3}$ and $20.1 \mathrm{~cm}^{3}$ (mid-RT, b), and $23.2 \mathrm{~cm}^{3}$ and $20.2 \mathrm{~cm}^{3}$ (post-RT, c), respectively. T2 maps show that right and left parotid T2 values are $70.9 \mathrm{msec}$ and $65.8 \mathrm{msec}$ (pre-RT, d), $75.4 \mathrm{msec}$ and $75.1 \mathrm{msec}$ (mid-RT, e), and $75.8 \mathrm{msec}$ and $72.2 \mathrm{msec}$ (post-RT, f), respectively. FF maps show that right and left parotid FF values are $54.9 \%$ and $57.4 \%$ (pre-RT, g), 59.0\% and 57.3\% (mid-RT, h), and $45.9 \%$ and $50.4 \%$ (post-RT, i), respectively

Correlations between parotid T2 or FF values and parotid atrophy rate or mean radiation dose

As shown in Table 2, the only significant correlation existed between the change rate of parotid T2 value and parotid atrophy rate from pre-RT to post-RT $(\mathrm{r}=0.313$, $P=0.027)$.

\section{Correlations among parotid T2 or FF value, parotid} volume, and MR scan time point

Multiple linear regression analysis showed that parotid T2 value negatively correlated with parotid volume (standardized coefficient $[\mathrm{SC}]=-0.259, P=0.001$ ), but positively correlated with $\mathrm{MR}$ scan time point ( $\mathrm{SC}=$ $0.476, P<0.001)$ significantly. Parotid FF value negatively correlated with parotid volume significantly $(\mathrm{SC}=-0.320$, $P=0.014)$, yet did not correlate with MR scan time point $(\mathrm{SC}=-0.104, P=0.417)$.
Reproducibility of parotid T2 and FF value measurements The intra- and inter-oberver ICCs of parotid T2 and FF values were 0.939 (95\% confidence interval [CI], 0.9160.955 ) and 0.935 (95\% CI, 0.910-0.953) for T2 value, and 0.992 (95\% CI, 0.985-0.996) and 0.984 (95\% CI, 0.9720.990) for FF value, respectively.

\section{Discussion}

Parotid volume decreased significantly after radiotherapy with an atrophy rate of $27 \%$, which might be due to the loss of acinar cells [10]. Houweling et al. reported a mean parotid volume shrinkage of $27 \%$ in patients with oropharyngeal cancer 6 weeks after radiotherapy [17], which was consistent with our result.

Parotid T2 value continuously increased during radiotherapy, which can be explained by the continuous aggravation of tissue edema [10]. Houweling et al. also reported 
Table 2 Correlations between the change rates of parotid T2 or FF values and parotid atrophy rates or mean radiation dose during radiotherapy

\begin{tabular}{|c|c|c|c|c|}
\hline & \multicolumn{2}{|l|}{$\mathrm{R}_{V}$} & \multicolumn{2}{|c|}{ Mean radiation dose } \\
\hline & $r$ & $P$ value & $r$ & $P$ value \\
\hline \multicolumn{5}{|c|}{$\overline{\text { Mid-RT }}$} \\
\hline $\mathrm{R}_{\mathrm{T} 2}$ & 0.092 & 0.525 & 0.087 & 0.530 \\
\hline $\mathrm{R}_{\mathrm{FF}}$ & 0.038 & 0.820 & 0.121 & 0.591 \\
\hline \multicolumn{5}{|c|}{ Post-RT } \\
\hline $\mathrm{R}_{\mathrm{T} 2}$ & 0.313 & $0.027^{\mathrm{a}}$ & -0.106 & 0.444 \\
\hline $\mathrm{R}_{\mathrm{FF}}$ & -0.029 & 0.863 & -0.419 & 0.052 \\
\hline
\end{tabular}

Note: Pre-RT within 2 weeks before radiotherapy, mid-RT 5 weeks after the beginning of radiotherapy, post-RT 4 weeks after radiotherapy. $\mathrm{R}_{\mathrm{V}}, \mathrm{R}_{\mathrm{T} 2}$ and $\mathrm{R}_{\mathrm{FF}}$ are the change rates of parotid volume, $T 2$ value and fat fraction (FF) compared with pre-RT, respectively. ${ }^{a} P<0.05$ with Pearson's correlation test

a significant increase of parotid T2 signal intensity 6 weeks after radiotherapy [17], which was in line with our results. We also confirmed a positive correlation between the change rate of parotid T2 value and parotid atrophy rate from pre-RT to post-RT. A possible explanation is that the enlarged extracellular space due to the loss of acinar cells caused an accumulation of free water in parotid parenchyma [10]. It was reported that parotid shrinkage was significantly correlated with the decrease of saliva production in patients with head-and-neck cancer undergoing radiotherapy [18]. However, the correlation between the change rate of parotid $\mathrm{T} 2$ value and parotid atrophy rate was weak. A similar finding was obtained by a previous study, which also showed low correlations between changes in parotid IVIM MR parameters and parotid shrinkage $(\mathrm{r}=0.336$ and $P=0.023$ for $A D C ; \mathrm{r}=0.357$ and $P=0.018$ for ADClow; $\mathrm{r}=0.378$ and $P=0.012$ for $f$ ) [19]. Hence, such a low correlation indicated that the change of MR parameters in irradiated parotid glands might be involved with other factors such as heterogeneous distribution of parotid radiation dose and different radiation sensitivity of individual parotid gland.

The pre-RT parotid FF value of NPC patients (mean age, 47.8 years) was approximately $38.2 \%$ in this study. Kise et al. reported a mean parotid FF value of $29.4 \%$ in healthy volunteers (mean age, 37.6 years) at 3.0-T MR scanner, which was slightly lower than ours [13]. Chang et al. reported a positive correlation between parotid FF value and age in healthy adults [20], which might explain the difference between Kise et al.'s and our results.

Parotid FF value first increased and then decreased during radiotherapy. Multiple linear regression analysis revealed that early increase of FF value was mainly due to parotid atrophy from pre-RT to mid-RT. From midRT to post-RT, parotid volume remained unchanged, while glandular edema continuously aggravated. The FF value reflects the relative proton density ratio between fat signal and the sum of fat and water signals [15, 21], which might suggest that the decrease of parotid FF value was due to glandular edema from mid-RT to post-RT.

Parotid T2 and FF values showed excellent intra- and inter-observer reproducibility. Serai et al. reported an ICC of 0.992 for parotid FF value in healthy volunteers obtained from mDIXON Quant imaging at 3.0-T MR scanner [22], which was consistent with our findings.

Our study had several limitations. First, the sample size was relatively small, yet still larger than other investigations on radiation-induced parotid damage with MR imaging $[17,19]$. Second, parotid gland biopsy was not performed due to its invasiveness. Third, long-term follow-ups was not performed. Evaluation of late fatty replacement in irradiated parotid glands should be further investigated.

\section{Conclusions}

T2 mapping and mDIXON Quant imaging provided pathophysiological information of parotid gland during radiotherapy, which could serve as an objective and quantitative modality in evaluating early radiationinduced parotid damage and facilitate a timely adjustment of treatment scheme to alleviate the damage of parotid glands.

\section{Abbreviations}

Cl: Confidence interval; FF: Fat fraction; ICC: Intraclass correlation coefficient; IMRT: Intensity-modulated radiotherapy; mid-RT: 5 weeks after the beginning of radiotherapy; MR: Magnetic resonance; NPC: Nasopharyngeal carcinoma; post-RT: 4 weeks after radiotherapy; pre-RT: Within 2 weeks before radiotherapy

\section{Acknowledgments}

We would like to thank the Department of Radiology, Nanjing Drum Tower Hospital for providing the MR scanner.

\section{Funding}

This study has received funding by National Natural Science Foundation of China (81371516), Foundation of National Health and Family Planning Commission of China (W201306), Social Development Foundation of Jiangsu Province (BE2015605), Natural Science Foundation of Jiangsu Province (BK20150109), Research Project of Health and Family Planning Commission of Jiangsu Province (Q201508), Six Talent Peaks Project of Jiangsu Province (2015-WSN-079), and Key Project supported by Medical Science and technology development Foundation, Nanjing Department of Health (YKK15067).

\section{Availability of data and materials}

The dataset supporting the conclusions of this article is included within the article.

\section{Authors' contributions}

NZ, CC and XD analyzed the data and drafted the manuscript; NZ and CC made substantial contributions to the acquisition of data; JY provided the oncological support; WBC controlled the quality of MR examination and data analysis; JH and ZYZ contributed to the conception and design. XFY revised the manuscript. All authors read and approved the final manuscript.

Ethics approval and consent to participate

This study was approved by the institutional review board of Nanjing Drum Tower Hospital, and all patients offered the written informed consents. 


\section{Consent for publication}

Written informed consent for publication was obtained from the patients.

\section{Competing interests}

The authors declare that they have no competing interests.

\section{Publisher's Note}

Springer Nature remains neutral with regard to jurisdictional claims in published maps and institutional affiliations.

\section{Author details}

'Department of Radiology, Nanjing Drum Tower Hospital, The Affiliated Hospital of Nanjing University Medical School, Nanjing 210008, China. ${ }^{2}$ Philips Healthcare, Shanghai 200233, China. ${ }^{3}$ The Comprehensive Cancer Centre of Nanjing Drum Tower Hospital, Clinical College of Nanjing Medical University, Nanjing 210008, China. ${ }^{4}$ Department of Radiation Oncology and Winship Cancer Institute, Emory University, Atlanta, GA 30322, USA.

Received: 17 October 2017 Accepted: 5 February 2018

Published online: 08 February 2018

\section{References}

1. de Castro GJ, Federico MH. Evaluation, prevention and management of radiotherapy induced xerostomia in head and neck cancer patients. Curr Opin Oncol. 2006;18:266-70.

2. Anand AK, Jain J, Negi PS, et al. Can dose reduction to one parotid gland prevent xerostomia? - a feasibility study for locally advanced head and neck cancer patients treated with intensity-modulated radiotherapy. Clin Oncol. 2006;18:497-504.

3. Konings AWT, Coppes RP, Vissink A. On the mechanism of salivary gland radiosensitivity. Int J Radiat Oncol Biol Phys. 2005;62:1187-94.

4. Kam MK, Leung SF, Zee B, et al. Prospective randomized study of intensitymodulated radiotherapy on salivary gland function in early-stage nasopharyngeal carcinoma patients. J Clin Oncol. 2007;25:4873-9.

5. Maes A, Weltens C, Flamen $P$, et al. Preservation of parotid function with uncomplicated conformal radiotherapy. Radiother Oncol. 2002;63:203-11.

6. Cox JD, Stetz J, Pajak TF. Toxicity criteria of the radiation therapy oncology group (RTOG) and the European Organization for Research and Treatment of cancer (EORTC). Int J Radiat Oncol Biol Phys. 1995;31:1341-6.

7. Konings AWT, Faber $\mathrm{H}$, Cotteleer $\mathrm{F}$, et al. Secondary radiation damage as the main cause for unexpected volume effects: a histopathologic study of the parotid gland. Int J Radiat Oncol Biol Phys. 2006;64:98-105.

8. van Acker F, Flamen P, Lambin P, et al. The utility of SPECT in determining the relationship between radiation dose and salivary gland dysfunction after radiotherapy. Nucl Med Commun. 2001;22:225-31.

9. Traxler M, Hajek P, Solar P, et al. Magnetic resonance in lesions of the parotid gland. Int J Oral Maxillofac Surg. 1991;20:170-4.

10. Stephens LC, King GK, Peters $L$, et al. Acute and late radiation injury in rhesus monkey parotid glands. Evidence of interphase cell death. Am J Pathol. 1986;124:469-78.

11. Tartaglino LM, Rao VM, Markiewicz DA. Imaging of radiation changes in the head and neck. Semin Roentgenol. 1994;29:81-91.

12. Perea Palazón RJ, Solé Arqués M, Prat González S, et al. Parametric methods for characterizing myocardial tissue by magnetic resonance imaging (part 2): T2 mapping. Australas Radiol. 2015;57:471-9.

13. Kise $Y$, Chikui T, Yamashita $Y$, et al. Clinical usefulness of the mDIXON quant the method for estimation of the salivary gland fat fraction: comparison with MR spectroscopy. Br J Radiol. 2017:90:20160704.

14. Mascaro L, Duina A, Grazioli L. Characterization of parotid gland tissue: a description of an MRI protocol set-up and results of in-vivo applications. Magn Reson Imaging. 1995;13:531-44.

15. Chang HC, Juan $\mathrm{CJ}$, Chiu HC, et al. Parotid fat contents in healthy subjects evaluated with iterative decomposition with echo asymmetry and least squares fat-water separation. Radiology. 2013;267:918-23.

16. Zhou N, Chen W, Pan X, et al. Early evaluation of radiation-induced parotid damage with diffusion kurtosis imaging: a preliminary study. Acta Radiol. 2017; https://doi.org/10.1177/0284185117710051.

17. Houweling AC, Schakel T, van den Berg CA, et al. MRI to quantify early radiationinduced changes in the salivary glands. Radiother Oncol. 2011;100:386-9.
18. Teshima K, Murakami R, Tomitaka E, et al. Radiation-induced parotid gland changes in oral cancer patients: correlation between parotid volume and saliva production. Jpn J Clin Oncol. 2009;40:42-6.

19. Marzi S, Forina C, Marucci L, et al. Early radiation-induced changes evaluated by intravoxel incoherent motion in the major salivary glands. J Magn Reson Imaging. 2015;41:974-82.

20. Chang HC, Juan CJ, Chiu HC, et al. Effects of gender, age, and body mass index on fat contents and apparent diffusion coefficients in healthy parotid glands: an MRI evaluation. Eur Radiol. 2014;24:2069-76.

21. Li G, Xu Z, Yuan W, et al. Short- and midterm reproducibility of marrow fat measurements using mDixon imaging in healthy postmenopausal women. Skelet Radiol. 2016;45:1385-90.

22. Serai SD, Dillman JR, Trout AT. Proton density fat fraction measurements at 1.5- and 3-T hepatic MR imaging: same-day agreement among readers and across two imager manufacturers. Radiology. 2017;284:244-54.

\section{Submit your next manuscript to BioMed Central and we will help you at every step:}

- We accept pre-submission inquiries

- Our selector tool helps you to find the most relevant journal

- We provide round the clock customer support

- Convenient online submission

- Thorough peer review

- Inclusion in PubMed and all major indexing services

- Maximum visibility for your research

Submit your manuscript at www.biomedcentral.com/submit
) Biomed Central 